\title{
Das literarische Problem des Hebräerbriefes.
}

Von E. Burggaller in GroB-Strehlitz, O.-Schl.

Der im 1906 Jahre verstorbene Professor Wrede in Breslau hat als sein letztes Werk nicht lange vor seinem Tode eine Untersuchung über "Das literarische Rätsel des Hebräerbriefes" erscheinen lassen . Es trägt gleich seinen vorher erschienenen Arbeiten deutlich die Eigenart dieses $z u$ früh abgerufenen Forschers an sich. Wr. war in mancher Beziehung ein einsamer Mann. Er liebte es, seine eigenen Wege zu gehen; sein scharfer kritischer Geist hinderte ihn, auf betretenen Bahnen zu wandeln. Innere Wahrhaftigkeit nötigte ihn, auch was allgemein festzustehen schien, auch was der unbefangensten Wissenschaft als unanfechtbar galt, doch noch einmal zu prüfen und zu untersuchen, ob nicht darin noch ein ungelöstes Problem enthalten sei. So hat er der Theologie, besonders in der Erforschung des Urchristentums neue Anstöße gegeben und ihren Blick auf ungeklärte, ja kaum beachtete Fragen gelenkt. Freilich, daß die Ergebnisse seiner Untersuchungen zu allgemein anerkannten Resultaten der Wissenschaft werden können, wird man bezweifeln dürfen. Es lag zwar etwas Bestechendes in der Art seiner Beweisführung, wie seiner ganzen Methode, wenn er es verschmähte, mit den hergebrachten Mitteln theologischer Arbeit an seinen Gegenstand zu gehen und die herkömmlichen Kategorien und Begriffe an ihn anzulegen. Die Wahrscheinlichkeitsrechnung der historischen Kritik genügte ihm nicht. Er suchte tiefer zu dringen, indem er die Möglichkeit einer Hypothese oder eines traditionellen Axioms nicht bloß an den mühsam zusammengesuchten Andeutungen einzelner Stellen und Worte prüfte, sondern sie mit dem ganzen Vorstellungsbilde, das wir uns von jener Zeit im ganzen oder von einzelnen Verhältnissen machen müssen, in Verbindung brachte und auf diesem Hintergrunde ihre Denkbarkeit

I In den „Forschungen zur Religion und Literatur des Alten und Neuen Testaments" von Bousset und Gunkel. 8. Heft. Göttingen, 1906. 
zu prüfen suchte. Aber oft genug verleitete ihn dies Bestreben dazu, statt des objektiven Maßstabs die eigne Empfindung fur das Gewicht seiner oder fremder Gründe bestimmen $\mathrm{zu}$ lassenx. Manche seiner Deduktionen, so einleuchtend sie sich zuerst lesen, erwecken doch den Verdacht, als ob Wr. unbewußt nicht grade sich von seiner vorgefaßten Meinung leiten lieb, aber als ob er da Probleme suchte, wo ein andrer sie beim besten Willen nicht mehr finden konnte, und daß er sich dadurch wie in einem Irrgarten verlor. Auch sein angeführtes letztes Werk trägt diese seine Art an sich.

Wer Wr. kannte, weib aber, wie sehr sachlicher Widerspruch ihm gefiel. Er war für jeden Versuch, seine Aufstellungen zu widerlegen, dankbar. In diesem Sinne seien auch die nachstehenden Ausführungen, die grade in den wesentlichen Punkten zu anderen Resultaten kommen, als er, seinem Andenken gewidmet.

Wr. geht von der richtigen Beobachtung aus, daß im Hebr. der Briefcharakter erst ganz am Schluß zu Tage tritt. Diesen Schluß, also etwa I3, I8-25 trennt er vom Hauptteil und behandelt beide gesondert. Schon hier dürfte die Frage naheliegen, ob die Methode berechtigt ist. Denn durch die Ausscheidung des brieflichen Schlusses nimmt Wr. vorweg, was er erst beweisen will. Wenn man aus einem Briefe alles, was seine Eigenschaft als solchen bestätigt und bekundet, ausschneidet, dann ist es freilich ein leichtes, von dem Reste zu behaupten, daß er nicht mehr den Eindruck eines Briefes mache. Doch beruht hierin nicht das Eigentümliche der Hypothese Wr's. Auch schon von früheren Forschern ist dem Hebr. die Eigenschaft des Briefes abgesprochen worden, - er nennt u. A. De Wette, Leonh. Hug, E. Reuß und vor allem Overbeck, dem er die stärkste Anregung verdankt. Während die meisten in 13, 18-25 einen Nachtrag späterer Hand erblicken wollen, durch den der vorangehenden systematischen Abhandlung der Briefcharakter aufgedrückt worden ist, hält Wr. an der Identität des Verfassers beider Teile fest. Auch mit dieser Ansicht steht er nicht allein. Reuss, v. Hofmann, Blass haben sie vertreten; sie haben sich aber die Sache.so erklärt, daß der Verf. zuerst allerdings nur einen

x Bezeichnend für diese seine Methode ist, was er in einem Briefe gelegentlich schrieb: „ich kann die Dinge nicht anders sehen." 
Aufsatz habe schreiben wollen, dann aber - aus irgend welchen Gründen - beschlossen habe, seine Schrift an bestimmte Leute zu senden. Dicser Gedanke erscheint Wr. undurclführbar. (S. 64.) Ihm ist es undenkbar, daß ein Schriftsteller „einen Brief hätte schreiben können, von dem er gar nicht gewulit habe, clab er ein Brief werden solle." Wr. hat nicht gemerkt, daß er mit diesem Argument das Recht seiner eigenen Methode untergraben hat. Den eben Genannten gegenüber will er daran festhalten, dab, wenn das Werk schlieblich vom Verf. als Brief benutzt, versandt worden ist, auch alle in ihm schon vorher enthaltenen Anreden, von 3, $\mathrm{I}$ an, auf die Absicht, einen Brief $z \mathrm{u}$ schreiben, schlielien lassen. Aber es ist nicht cinzusehen, warum dieser Einwand nicht auch seiner Hypothese, daß der Verfasser des Ganzen erst zum Schlub die Absicht einer Fiktion gefabt habe, entgegengehalten werden kann. Der Briefschluß, sagt er, stimme nur dann mit der nichtbrieflichen Haltung des Schriftstücks, wenn ersterer "Schein und Täuschung“ ist (S. 65); und noch deutlicher S. 70: Die Schrift war fertig, an ihr selbst hatte er nichts $z u$ ändern, - da verfiel der Verfasser, um ihr einen besseren Eingang zu verschaffen, „am Ende auf den Gedanken der Fiktion“; er entschlols sich, um seiner Schrift Leser und Anerkennung zu gewinnen, ihr den Anschein eines Briefes, genauer eines paulinischen Gemeindebriefes zu geben. $\mathrm{Da} b$ sich mit dieser Annahme eine ganze Reihe von Schwierigkeiten ergeben, hat Wr. selbst gefühlt. Über den Einwand, $\mathrm{da} ß$ man dann von dem Verfasser auch die nachträgliche Hinzufügung eines Briefeingangs oder wenigstens einer Anrede erwarten mübte, hilft er sich mit der Erklärung, daß es am wahrscheinlichsten sei, der Verfasser sei auf den uns so naheliegenden Gedanken einer Ergänzung des Anfangs gar nicht gekommen (70); für die merkwürdige Tatsache, daß die Hindeutungen auf Paulus am Schlusse, d. h. in dem fingierten Schlusse nicht massiver ausgefallen sind, hat er überhaupt keine weitere Erklärung. Und doch scheint mir dies letzte Moment von ganz wesentlicher Bedeutung. Denn genau genommen, sind es nur 4 Verse im letzten Cap., durch die jene Fiktion zu schaffen versucht wvorden wäre: 19, 22, 23, 24; und die paulinische Abfassung wäre gar nur in 23 und höchstens noch in $24^{\mathrm{b}}$ of åmò $\tau \hat{\eta} c$ 'I $\tau \alpha \lambda i$ ac angedeutet. Allerdings für einen Schriftsteller, der mit vollem Bewußtsein den Anschein erwecken wollte, daß nicht er, sondern ein andrer die vorangehende, im Vergleich zu diesen Schlußversen umfangreiche Abhandlung geschrieben und einer bestimmten Gemeinde zugesandt habe, eine merkwürdige und schwer begreifliche Bescheidung. 
Aber noch unbefriedigender ist Wr's Antwort auf die Frage: was denn eigentlich überhaupt von Anfang an die Absicht des Schreibers gewesen ist, wenn er erst ganz am Ende den Entschlub gefabt hat, sein Werk als einen Brief zu veröffentlichen? Wr. erkennt die Berechtigung dieser Frage an, wenn er S. 33 sagt: „Es scheint aber doch, als ob der Autor selbst sich beim Schreiben in einer bestimmten Rolle gefühlt haben müsse, entweder in der des Redners (Homileten) oder des Briefschreibers." Sollte es wirklich nur so scheinen? Es muß so gewesen sein. Irgend eine Absicht muß er von Anfang an gehabt haben. Es setzt sich doch keiner hin, bloß um zu schreiben; wenigstens werden wir sicherlich einem Christen jener Zeit nicht zumuten wollen, daß er ohne Absehen auf seine Glaubensgenossen (oder auf solche, die er gewinnen wollte), einfach eine Abhandlung über die ihn bewegenden religiösen Probleme hätte schreiben wollen. Das will auch Wr. nicht behaupten. Er muß zugeben, daß der Verf. mindestens von 3,1 an irgend ein, wenn auch noch so unbestimmtes, ideales Publikum vor Augen gehabt habe. Die als Abhandlung begonnene Schrift setzt sich demnach bald als ein Brief fort und endet als ein paulinischer Gemeindebrief (73). Also muß man sich nach Wr. die Entstehung von Hebr. etwa so denken: ein christlicher Lelurer fühlt sich gedrungen ein ihn interessierendes Thema in einer Abhandlung zu erörtern; erst beim Schreiben kommt ihm der Gedanke an das Publikum, das seine Schrift lesen wird; er redet es an, teils unwillkürlich, teils mit voller Absicht rhetorische Kunstgriffe benutzend, um sein Interesse zu erregen, (z. B.) 5, II ff.); aber er denkt dabei weder an eine bestimmte Gemeinde, noch überhaupt an einen irgendwie begrenzten Kreis innerhalb der damaligen Christenheit; erst, als er fertig ist, beginnt er zu überlegen, wie er seinem Werke am leichtesten Eingang verschaffen könnte, und da entschließt er sich, ihm durch einige angehängte, im letzten Grunde recht mißverständliche Andeutungen den Anschein eines paulinischen Gemeindebriefes zu geben. Aber was diesem Anonymus dadurch, da $\$$ er die wenigen Verse am Schluß hinzugefügt hat, gelungen ist, nämlich einen Brief zustande zü bringen, von dem er anfangs gar nicht gewußt hat, daß „es ein Brief werden soll", das mübte man auch jedem andern zugestehen, der den Schluß nicht hinzugefügt hätte, um seine Leser über den Ursprung seines Werkes zu täuschen, sondern der ganz unbefangen seine zuerst nicht als Brief gedachte Schrift als einen solchen d. h. in Briefform der Öffentlichkeit zu übergeben, nachträglich beschloß. Das, was Wr. selbst als das eigentlich Neue seiner Hypothese bezeichnet, - die nach 
Fertigstcllung der Abhandlung versuchte Fiktion eines paulinischen Bricfes, - ist in Wahrheit ebenso schwer denkbar, als die von ihm abgelchnte Annahme Reuss' u. a. Man könnte wohl zugcben, daß eine ganz unpersönlich gehaltene, keine Anrede, keinerlei Beziehung zu bestimmten oder unbestimmten Lesern enthaltende Abhandlung mit einem Anhang versehen und dann als Brief versandt oder veröffentlicht worden sein könnte. Hat aber der Verfasser an Leser sciner Schrift, auf die er wirken wollte, gedacht, sie angeredet, ja, um ihre Aufmerksamkeit und ihr Interesse zu erregen, rhetorische Kunstgriffe angewandt ( 37 f.), dann ist es in beiden Fällen in gleichem Maße unwahrscheinlich, daß er sich von dem, was seine Schrift eigentlich werden sollte, keine Vorstellung gemacht habe.

Scheint auch die neue Kombination Wr's der Lösung des literarischen Problems des Hebr. Br. nicht näher zu führen, so ist es doch sein Verdienst, noch einmal die Gründe, die gegen den Charakter als Brief im eigentlichen Sinne, als Gemeindebrief sprechen, auf ihre Bedeutung untersucht und in ihrem starken Gewicht gewürdigt zu haben. Es kommen vor allem drei Punkte in Betracht, für die von den Auslegern, die an der traditionellen Auffassung, daß Hebr. ein an eine bestimmte Gemeinde oder an einen irgendwie begrenzten Kreis gerichteter Brief sei, festhalten, keine genügende Erklärung gefunden werden konnte; was gesagt wurde, ist immer wieder angefochten und durch neue Annahmen ersetzt worden.

I. Ein konkreter Anlab, der zu seiner Abfassung geführt hätte, läßt sịch aus Hebr. nicht erweisen. Werden auch die längeren, lebhaften Partieen von kürzeren Ermahnungen unterbrochen, so sind diese doch so wenig einheitlich, da $\mathrm{b}$ es nicht besondere Umstände oder Ereignisse gewesen sein können, die den Verfasser veranlaßt hätten, sich zum Trost, zur Ermahnung oder zur Ermunterung an seine Leser zu wenden.

2. Wie ungenügend und gezwungen alle Erklärungen für das Fellen der Grußüberschrift sind, hat Wr. treffend nachgewiesen. Es handelt sich dabei stets um Mutmaßungen, die je nach Anschauung und Temperament dem einen mehr, dem andern weniger wahrscheinlich vorkommen, für die aber ein wirklicher Anhalt in Hebr. in keinem Falle geboten wird.

3. Es sind nur einige wenige Verse, die dem Schriftstück den 
Charakter eines Briefes zu geben scheinen: 13, 19; 22-24, und grade sie wollen zu dem übrigen Inhalt nicht recht stimmen. Der Verf. stellt seinen Lesern seine baldige persönliche Ankunft in Aussicht. (13,23 und nach der gewöhnlichen Auslegung auch V. 19). Die Frage ist immer wieder geäußert worden: wozu dann die lange Erörterung? Nur um sie in einige Mahnungen zur Treue und zum Ausharren ausklingen zu lassen? So wenig sie dem Verf. nebensächlich sind, machen sie doch auch keineswegs den Eindruck, als ob sie ihm so wichtig gewesen seien, daß er mit ihnen nicht bis zu seiner Ankunft hätte warten können.

Soweit besteht Wr's Behauptung zu Recht. Die angeführten Gründe werden der Auffassung, daß Hebr. ein an eine Orts- oder Hausgemeinde oder an einèn Kreis von Gemeinden gerichteter Brief sei, stets Schwierigkeiten bereiten. Allein das Fehlen eines konkreten Anlasses, sowie der Überschrift und Anrede und noch weniger das unter 3 angeführte schließen jede Beziehung des Verf. zu seinen Lesern oder genauer zu den von ihm angeredeten Personen aus. Zumeist ist wohl die Frage so gestellt worden: entweder ist Hebr. kein wirklicher Brief, dann können auch solche Beziehungen in ihm nicht gefunden werden, - oder sie sind vorhanden, dann muß es ein Brief sein. Wie aber, wenn trotz jener Bedenken gegen den Briefcharakter, deren Gewicht man freilich verschieden beurteilen, die man aber sicherlich nicht einfach beseitigen kann, nun doch nicht zu verkennen ist, daß der Verf. unter denen, an die er seine Worte richtet, sich Christen denkt, die ihm, wenn nicht der Person nach, so doch nach ihren Verhältnissen bekannt sind? Die Gefahr, jene Alternative als feststehend und unangreifbar anzusehen und darum dem klaren Wortlaut des Textes Gewalt anzutun, scheint mir auch $W r$. nicht entgangen zu sein. Er will auch nicht die geringste persönliche Beziehung des Verf. zu seinen Lesern finden. Alles, was auf eine solche hindeutet, sind in Wahrheit nur Redeformen, absichtlich gewählte, rhetorische Floskeln, aus denen man, durch jene Annahme, einen wirklichen Brief vor sich $z u$ haben, irregeleitet, etwas auf ein näheres Verhältniṣ zwischen Verf. und Lesern Hinweisendes herausgelesen hat. Allerdings meint Wr. einmal (S. 29): er habe „seire Umgebung als Publikum gedacht, nur natürlich eine weitere und ihrer Grenzen nach unbestimmte Umgebung“, vielleicht (ebenda Anm.) eine solche, „daß der Gedanke, sie auf dem Wege eines wirklichen Briefes zu erreichen, unmöglich wird“. Es könnte ja auch sein, daß die besondern Verhältnisse seiner Umgebung, ihre Erfahrungen, Befürchtungen und Mängel dem Verfasser einzelne seiner Mahnungen nahegelegt oder auch, ohne daß dies deutlich 
in sein Bewußtsein trat, ihn bei der Formulierung derselben becinflubt hätten. Aber in letzten Grunde ist es doch Wr's Mcinung, daß der Verfasser sich keine bestimmte Vorstellung von denen, die sein Geisteswerk genießen werden, gemacht hat. $\mathrm{Dab}$ Namen und Personalien fehlen, wird dabci noch nicht das wichtigste sein. (Im Galaterbriefe ist z. B. auch kcin Name enthalten, der von einer nähcren Bekanntschaft des Apostels mit den Gemeinden, an die er schrieb, Zeugnis ablegte.) Aber nach Wr. müßte der Verfasser eine sonderbare Gleichgiltigkeit der Frage gegenüber, auf wen er denn mit seinen Erörterungen und Mahnungen wirken würde, gehabt haben; er soll gewisse rednerische Wendungen und Figụren (z. B. 6, 9) in der Erwägung gewählt haben: wer immer sein Leser werden mag, der wird darin etwas Ermunterndes und Ermutigendes finden. So hat er wohl Mahnungen ausgesprochen, die "bestimmte Beobachtungen und Erfahrungen voraussetzen", die sich „aus der großen Forderung des Tages, weil angesichts der Lage Verzagtheit, Erschlaffung und Unsicherheit um sich zu greifen" drohten, von selbst ergaben, aber nicht solche, die „eine aus persönlichen Bekannten bestehende Leserschaft" forderten (34). Daher bleiben die Beziehungen des Verfassers zu den Lesern schattenhafte (13), die Schilderung ihrer Verhältnisse farblos; sie läbt sich nur dann verstehen, wenn man erkennt, daß der Kreis, an den er schreibt, überhaupt kein „Kreis“ mit festen Grenzen war. (2I.) Dem Eindruck, daß der Brief einige Andeutungen enthält, die eine persönliche Beziehung zwischen Schreiber und Leser fordern, hat sich allerdings auch $W_{r}$. nicht entziehen können. Er versucht die wenigen Stellen, die darauf hinzuweisen scheinen, zu allgemeinen, für alle Gemeinden jener Zeit passenden Mahnungen zu wandeln. Bei 10, 25 könnte er Recht haben. Käme in einer heute nicht wirklich gehaltenen, - sondern für den Druck geschriebenen Predigt eine Klage über schlechten Kirchenbesuch vor, so wäre es ein falscher Schluß, daraus zu folgern, daß sie für eine bestimmte einzelne Gemeinde berechnet gewesen sei. Aber anders steht es doch bei den Abschnitten 10, 32-34 und 12, 4 ff. und vor allem bei der die Abhandlung unterbrechenden Digression in $5,11-6,12$. In den erstgenannten Versen handelt es sich um die Erinnerung an frühere Verfolgungszeiten. Hier hat Wr. sicherlich das Gewicht aller einzelnen Züge zusammen zu gering angeschlagen. Wenn der Verfasser ihnen ins Gedächtnis zurückruft, wie sie nicht bloß geschmäht worden sind, sondern noch andere Drangsale erfahren haben, wie sie unter den Verfolgungen andrer mitgelitten und die Konfiskation ihres Vermögens in Geduld ertragen haben, 
und wenn er sie ermuntert, ihre Zuversicht nicht wegzuwerfen, und sie darauf hinweist, dab es bei ihnen ja noch nicht zum Blutvergießen gekommen sei, dann wird es doch schwer, sich vorzustellen, dab damit auch nur an ein ,ideales Publikum" gedacht sein solle. Auch dann würde das gelten, wenn wir wirklich von vielen Verfolgungen in den letzten Jahrzehnten des ersten Jahrhunderts nichts wiißten; nur wirkliche allgemeine Verfolgungszeiten, die mehr oder weniger die junge Christenheit betroffen hätten, konnten den Verfasser berechtigen, so zu einem idealen Publikum zu sprechen. Von solchen aber-müßten wir etwas wissen, auch wenn uns einzelne Bedruickungen durch Statthalter und Behörden unbekannt geblieben sind. Kommen, wenn die neronische Verfolgung nach Wr's Meinung wirklich unbedingt abzulehnen wäre, nur solche in Betracht, dann bleibt erst recht nur die Annahme, daß der Verfasser, wenn nicht eine Ortsgemeinde, so doch sicher einen bestimmten Kreis von Gemeinden im Auge gehabt haben könne.

Noch weniger kann es gelingen, die Beziehungen des Verfassers zu seinen Lesern aus dem Abschnitt 5, I I-6, I2 herauszudeuten. Denn das kann doch nicht im Ernst als ein Argument gegen sie betrachtet werden, $\mathrm{da} b$ in den christlichen Gemeinden jener Zeit überall "einer Minorität von Führenden eine Majorität von Geführten gegenüber stand" (32) d. h. daß es in allen Gemeinden Unreife gab, die an der Zeit ihrer Zugehörigkeit zur Gemeinde gemessen in ihrer Erkenntnis schon hätten weiter fortgeschritten sein müssen. Hier ließe sich der obige Vergleich in umgekehrtem Sinne anwenden: man dürfte bei einer heutigen Predigt doch nicht aus dem Umstande, daß bestimmte Mahnungen in ihr auch für die verschiedensten Gemeinden in einem weiten Gebiete gelten, folgern, daß sie nicht ursprünglich für eine einzelne, ganz bestinmte Gemeinde berechnet sein könnte. Wr. will dem Verfasser eine Inkonsequenz nachweisen, weil er seine Leser zuerst für die schwierige Frage, die er behandeln will, nicht reif hält, dann aber sich in ihrer weiteren Erörterung nicht stören läßt, und darum in dem ganzen Abschnitt 5, 11-6, 12 nur einen rhetorischen Kunstgriff sehen, - nur dazu bestimmt, das Interesse seiner Leser auf sein Thema zu lenken. Aber er übersieht, daß die Abschweifung nach 5, II den $Z$ week hat, die Ausführlichkeit, nicht die Schwierigkeit der nachfolgenden Darlegung zu erklären oder, wie v. Soden sagt (H. K. 3 Seite 46 ) ihn gegen den Verdacht zu sichern, als ob er nur aus "Redelust" ausführlich werde; „nur in eurem Interesse und mit Rücksicht auf eure Art bin ich ausführlich, nur eure Schwäche macht die Klarlegung schwierig“. Be- 
achtet man dies, dann ișt das Verfahren keineswegs mehr inkonsequent oder von Mangel an seelsorgerlichem Takte zeugend; es war ganz natürlich, daß der Verfasser von jener einen Tadel enthaltenden Bemerkung über die Reife seiner Leser zu einer Erwähnung ihrer Bedürfnisse, des für sie angemessenen Lehrstoffes und dann $\mathrm{zu}$ einer begütigenden und ermunternden Einschränkung seiner Vorhaltung geführt wurde. Dann fállt aber jeder Grund, eine Beziehung zu seinen Lesern in diesen Worten durchaus nicht anzuerkennen, fort. Vielmehr mübte man dem Schriftsteller, der - nur ein ideales Publikum vor Augen - mit seiner Abschweifung die Aufmerksamkeit der Leser zu erregen beabsichtigte, den Vorwurf machen, daß er dies in einer möglichst ungeschickten Weise getan hätte. Er hätte mit diesem Ziele vor sich viel eher versuchen müssen, einen der in dem Abschnitte ausgesprochenen Gedanken mit aller Schärfe und Deutlichkeit zu entwickelnl Er wäre entweder innerlich dazu genötigt worden, aus seinem Tade. die nachdrückliche Forderung $z u$ angespannter Aufmarksamkeit $z u$ folgern, oder es hätte sich ihm von selbst ergeben, grade hier einen Grund anzuführen, warum er sein Thema für so wichtig hält, dab er seine Behandlung nicht länger verschieben wolle. Statt dessen aber stellt er mehrere persönliche Bemerkungen nebeneinander: er muß ausführlich reden und sich Mühe geben, um verständlich zu werden, weil seinen Lesern das nötige Verständnis fehle; er wolle aber von den Anfangsgründen ein andres Mal zu ihnen reden, (man beachte auch dieses für ein ganz unbestimmtes Publikum sonderbare Versprechen); er hoffe von ihnen, daß sie nicht aus dem Glauben fallen und verloren gehen werden, und daß Gott sie, die zwar an Erkenntnis noch so schwach, aber an Liebe stark seien, nicht vergessen werde. $\mathrm{Zu}$ diesem Wechsel der Gedanken konnte nur veranlabt werden, wer bestimmte Leute vor sich sah, wem nicht das abstrakte Thema die Hauptsache war, während er diese Worte schrieb, sondern wem vor allem daran lag, diesen Christen, an die er dachte, gerecht zu werden und nach keiner Seite ihnen zu nahe zu treten. Somit scheint die These Wr's von dem „idealen Publikum“, für das Hebr. geschrieben wurde, unhaltbar zu sein.

Wenn nun aber einerseits die "Hebräerbrief" genannte Schrift unzweifelhaft nicht den Charakter eines wirklichen, aus bestimmtem Anlab verfa@ten Briefes an sich trägt, andrerseits dem Schriftsteller doch auch wieder ein bestimmter Kreis mit besonderen Erfahrungen und konkrete 
Verhältnisse vor Augen gestanden haben, dann ist sein Rätsel auch nicht gelöst, wenn man ihn als Epistel, als literarischen Brief bezeichnet. Das hat außer Wrede auch Deilmann in seinen Bibelstudien (1895) getan. Seine Unterscheidung zwischen wirklichem Brief und Kunstbrief, der nur die Form des Briefes benutzenden Abhandlung, für die er den Terminus Epistel vorschlägt, ist sicherlich voll berechtigt, aber dem Hebräerbriefe gegenüber $\mathrm{m}$. E. ohne Bedeutung. Denn grade das ist ja das bezeichnende an der Epistel, daß ihr Publikum ein ideales ist, das erst zu finden ihr überlassen bleiben mu円. Der Epistolograph schreibt eine Abhandlung, deren Leserkreis unter Umständen freilich auch irgendwie begrenzt sein kann, in den meisten Fällen sogar sein muß; er wendet sich auch an einen "Ausschnitt aus der unermeßlichen Öffentlichkeit" (Deißmann S. 245), aber immerhin an eine Öffentlichkeit, wenn auch an eine relative. Das Eigentümliche der Epistel ist eben die „ideale Unbestimmtheit ihrer Bestimmung"; der allgemeine, wenig konkrete Inhalt ist erst eine Folge hiervon. Wenn aber, wie wir gesehen haben, bestimmte persönliche Beziehungen zwischen dem Schreiber und seinem Publikum nicht entfernt werden können, - es ist hierbei ziemlich irrelevant, ob man sie nur an den oben erwähnten oder vielleicht auch noch an einigen anderen Stellen findet ${ }^{x}$, - so fehlt unsrer Schrift das Charakteristikum der Epistel, des Kunstbriefes, der sich an ein ideales Publikum richtenden dozierenden Abhandlung.

Ist sie nun aber weder ein "wirklicher Brief", noch eine "wirkliche" oder eigentliche „Epistel", so bleibt nur der Ausweg, sie der Gattung der „niedergeschriebenen Rede“ zuzuweisen. Deißmann stellt, allerdings nicht bei der Besprechung des Hebr., sondern bei der des Jac. Briefes, der neuerdings öfters als eine Homilie bezeichnet worden sei, die Forderung auf, daß auch dieser Begriff einer ähnlichen Differenzierung unterzogen werde, wie er sie dem Begriffe „Brief" gegenüber versucht habe. Hier scheint aber die Entwicklung nicht so einleuchtend zu sein oder sich nicht so von selbst zu ergeben; die Sachlage ist komplizierter. Für den Brief hat Deißmann diese Entwickelungslinie aufgefunden: I. wirklicher Brief; 2. nachträglich zur Literatur gemachter Brief; 3. Epistel; 4 fingierte Epistel. Diese 4 Formen reduzieren sich aber in Wahrheit auf zwei, da der nachträglich herausgegebene und vervielfältigte Brief seinem Ursprunge nach immer ein wirklicher Brief bleibt, und der Unterschied zwischen Epistel und fingierter Epistel, da jene 
selbst stets eine Fiktion ist, mehr oder weniger verschwindet. Mithin vereinfaclit sich der uns überlieferten Briefliteratur gegenüber das Problem zu der Frage, ob wirklicher oder Kunstbrief, Epistel? Die literarischen Möglichkciten, dic sich aus dem Begriff der Rede ergeben, können zu viel verwickelteren Kombinationen führen. Vor allem ist dies festzuhalten: wälırend der Brief, auch der wirkliche oder präliterarische, doch eben mit allen Litcraturwerken dies gemeinsam hat, daß er gleichzeitig mit seiner Entstehung ein Schriftwerk wird, ist bei der Rede die Möglichkeit vorhanden, daß sie vor ihrem eigentlichen Dasein als Rede oder hinterher niedergeschrieben sein kann. Selbstverständlich interessiert sie uns hier nur soweit, als sie wirklich niedergeschrieben wird; die nur mündlich gehaltene, extemporierte, weder vom Redner, noch von einem seiner Zuhörer fixierte Rede scheidet aus unserer Untersuchung völlig aụs. Aber die Unterscheidung zwischen vorher und nachher niedergeschriebener Rede ist nicht nebensächlich; denn es ist klar, da@ sich die vorher konzipierte Rede, auch wenn sie nur für einen einzelnen Fall bestimmt gewesen ist, viel mehr der Art andrer literarischer Werke nähern wird, als die erst später, aus welcher Veranlassung und mit welcher Bestimmung es auch sei, aufgezeichnete Rede. Ferner läbt sich das Merkmal des "wirklichen Briefes", daß er nicht wiederholbar sei, nicht ohne weiteres auf den Begriff der "wirklichen Rede" anwenden. Treten wir an irgend ein Schriftwerk der Vergangenheit mit der Frage heran, ob es etwa ursprünglich eine Rede war oder doch wenigstens zuerst zum mündlichen Vortrag bestimmt gewesen ist, dann ist die Beobachtung, daß sein Inhalt allgemeiner Art ist und konkreter Beziehungen entbehrt, für die Beantwortung unsrer Frage ohne wesentliche Bedeutung. Das, was den wirklichen Brief konstituiert, hat für die Beurteilung und Anerkennung einer niedergeschriebenen Rede keinen Wert. Gewi die Reden des Demosthenes gegen Philipp, die Reden Bismarcks im deutschen Reichstage tragen die Zeichen ihrer ursprünglichen Bestimmung und ihres Charakters deutlich an sich; sie sind unwiederholbar. Aber wenn ein Nationalökonom in einem politischen Vereine einen Vortrag über Freihandel und Schutzzoll hält, ein Forschungsreisender die Verhältnisse einer Kolonie schildert, so braucht in ihren Ausführungen keine Andeutung einer persönlichen Beziehung zu ihren Zuhörern vorzukommen; was sie sagen, könnte an Ioo anderen Orten, vielleicht auch noch nach Jahren mit dem gleichen Rechte vorgetragen werden; und doch wäre es falsch, es darum für unmöglich zu halten, daß nicht ein ganz besonderer Anlaß und die Rücksicht auf ein bestimmtes Ziel jene 
Reden hervorgerufen habe. Was Deißmann (S. 218) vom Briefe sagt, gilt auch von der Rede: „für die Beantwortung der Frage, wo wir wirkliche Briefe vor uns haben, kann niemals die Form entscheidend sein, sondern in letzter Linie nur die Absicht des Verfassers; es sollte zwar nicht sein, aber es kann doch Briefe geben, die sich lesen, wie ein Libell. ..." Endlich ist noch zu beachten, daß auch der Begriff der fingierten Rede kein einheitlicher ist; die fingierten Reden bei den Geschichtsschreibern des Altertums oder die im Johannes-Evangelium, also Reden, die geschichtlichen Persönlichkeiten in bestimmten Situationen in den Mund gelegt und zumeist in die Erzählung eingefügt werden, stellen eine ganz andere Literaturgattung dar, als etwa Fichtes Reden an die deutsche Nation oder Schleiermachers Reden über die Religion. Die letzteren gehören wohl im besondern in das Gebiet, das Deißmann als das der "literarischen Rede" bezeichnet (S. 242); sie haben (oft noch weniger als die Epistel mit dem wirklichen Briefe,) mit der wirklichen Rede nur die Form gemeinsam; oder der Schriftsteller hat für sein Werk diesen Namen gewählt, um den lebendigeren Stil und die kräftigeren Töne, die er anschlägt, zu rechtfertigen. So mancherlei Komplikationen sich aber auch ergeben, die Hauptfrage bleibt doch die, ob ein uns überliefertes Schriftwerk als Wiedergabe einer einmal oder mehrere Male wirklich gehaltenen Rede anzusehen ist oder nicht? In dem ersteren Falle bleiben zwei Wege offen: entweder das gesprochene Wort war das primäre, ursprüngliche, - das wäre die eigentliche, wirkliche Rede, - oder die Schrift war zuerst da und der mündliche Vortrag folgte, aber natürlich als Hauptzweck. Es braucht nicht genauer ausgeführt zu werden, da $\$$ die Verlesung an sich den literarischen Charakter eines Werkes nicht verändert; den neutestamentlichen Schriften ist ja wohl fast ausnahmslos dieses Schicksal zuteil geworden. Aber jene Unterscheidung muf gemacht werden, um eben die ursprünglich zum mündlichen Vortrag bestimmte Schrift von dem eigentlichen Literaturwerk, das hernach auch zur Verlesung verwendet worden ist, zu scheiden.

Wenn nun Wrede (S. 26), die 10, 32 ff. vom Verfasser benutzte Gegenüberstellung von Gegenwart und Vergangenheit ein wirksames homiletisches Motiv nennt, und die Möglichkeit zugibt, daß er es auch einmal in mündlicher Predigt vor einer Einzelgemeinde verwertet haben könnte, so hat er damit eine ganz richtige Beobachtung ausgesprochen, aber er hat diesen Gedanken nicht weiter verfolgt, weil für ihn der Begriff der nachträglich niedergeschriebenen Rede überhaupt 
nicht in Betracht kam; der Schlub, der den Anschein cincs Briefes erwecken solltc, war ihm Beweis genug, dab auch das ganze Werk nur eine fingierte Epistel sein könne. Auch sonst finden sich in Wr's Abhandlung eine ganze Anzahl von Zeugnissen dafür, daß er sich dem Eindruck, unsre Schrift erinnere an eine nachträglich niedergeschriebene Rede, nicht entziehen konnte, dall dieser Gedanke sich ihm immer wieder aufdrängte. So sagt er (S. 21): „Ohne auf diesen Punkt ein ungebührliches Gewicht zu legen, darf man doch sagen: das "wir" entspricht mehr dem Prediger, der allgemein giltige Sätze und Vorschriften ausspricht, als dem Briefsteller..." An einer Stelle (S. 24) nennt er den Hebr.brief geradezu eine Predigt in Briefform und ein anders Mal (S. 73) eine Mischung von Abhandlung und Predigt. An der letzten Stelle sagt er weiter: „Es scheint aber doch, als ob der Autor selbst sich beim Schreiben in einer bestimmten Rolle gefühlt haben müsse, entweder in der des Redners (Homileten) oder des Briefschreibers." Auch hier sieht man deutlich, daß Wr. sich die Entstehung unsrer Schrift nicht ohne eine bestimmte literarische Tendenz hat vorstellen

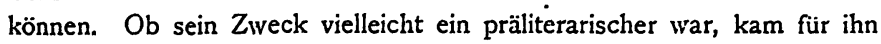
nach Ablehnung des wirklichen Briefcharakters nicht mehr in Frage. Ähnlich urteilt auch Deißmann (S. 242), der die Selbstbezeichnung in

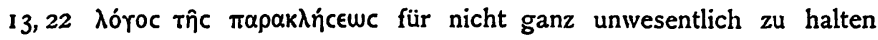
scheint und dann fortfährt: „man hätte gar keine Veranlassung, ihn (den Hebräerbrief) für etwas andres als für eine literarische Rede zu halten, also nicht einmal für eine Epistel," wenn nicht der Schluß die Vermutung nahelegte, daß der Briefeingang verloren gegangen sei. Das ist aber, wie Wr. (S. 7 f.) mit Recht nachweist, eine Ausflucht, die nur wenig Wahrscheinlichkeit für sich beanspruchen kann. Eine literarische Rede, d. h. wie ich D. verstehe, ein nur den Stil und die Lebendigkeit der Rede nachahmendes, sich aber an die Öffentlichkeit wendendes und sein Publikum erst suchendes Literaturwerk kann eben der Hebräerbrief nicht sein, wenn es, wie oben zu zeigen versucht wurde, unmöglich ist, zu leugnen, daß persönliche Beziehungen des Verfassers zu seinem Publikum und die Rücksicht auf bestimmte konkrete Verhältnisse in ihm zu finden sind. Am weitesten in der von uns eingeschlagenen Richtung ist v. Soden (K. H. C. S. 6-7) gegangen, der die Behauptung aufstellt, daß unsre Schrift nach den Regeln der griechischen Rhetorik aufgebaut sei. Hebr. ist "weniger als Brief gedacht und aufgesetzt, denn als ein schriftlicher Vortrag, vielleicht ein, wenn auch über das $\mathrm{Ma} b$ mündlicher Rede hinausgehendes Beispiel für die Art von Vorträgen, wie sie in den 
christlichen Versammlungen (wenigstens Roms) von hervorragenden Männern schon damals unter Umständen zu hören waren." Wie weit v. Sodens Behauptung, daß „der Aufbau des Briefes völlig den Gesetzen der antiken Rhetorik" entspreche, begründet oder wirklich so fragwürdig sei, wie Wr. meint (S. 37), kann hier nicht weiter untersucht werden. An und für sich ist nicht recht ersichtlich, warum ein christlicher Redner, - und der Verfasser des Hebr. Briefes macht unbestritten den Eindruck eines rhetorisch geschulten Mannes, - sich nicht der antiken Kunstformen sollte bedient haben, auch wenn dabei einige Abweichungen zu konstatieren wären. Andrerseits aber verstehe ich nicht, warum v. S. aus seiner Aufstellung nicht die Folgerung, da $\$$ uns eine christliche Rede vorliegt, gezogen hat? Liegt es nicht bei ihm, wie bei Wr. und D. daran, dal man im allgemeinen noch viel zu sehr gewohnt ist, jene Zeit der christlichen Gemeinde vor allem als eine literarisch produktive oder wenigstens die literarische Produktion als eine wesentlich selbständige Lebensäußerung anzusehen, während sie doch tatsächlich nur ein schwacher und verschwindend kleiner Niederschlag einer starken zur mündlichen Wirksamkeit und Propaganda, zum Zeugen und Bekennen anregenden und dazu fortreißenden Bewegung gewesen ist. (Vgl. I Kor 14 oder Jac 3.) Jene Vorstellung, als ob die ältesten christlichen Schriftsteller nach langer Meditation und mühsamer Durchforschung und Vergleichung schriftlicher Quellen und Vorbilder ihre Werke niedergeschrieben und komponiert hätten, ist in vielen Fällen, ob auch unbewußt, von größerem Einfluß, als es berechtigt ist. Was Deißmann von den Briefen des Paulus überzeugend nachgewiesen hat, nämlich daß sie eigentlich präliterarischen oder überhaupt unliterarischen Charakters sind, wird zum großen Teil auch von den andern neutestamentlichen Schriften, vielleicht sogar von den johanneischen, gelten müssen.

Sicher aber wird man es von dem Hebr. Briefe sagen müssen. Richtet in ihm ein christlicher Lehrer (oder Evangelist) an bestimmte Leute, deren Verhältnisse und Erfahrungen bekannt sind, Worte der Belehrung und Ermahnung, der Aufmunterung und Zusprache, so bleibt, wenn gegen den Charakter als wirklicher Brief wichtige Gründe sprechen, nur die Möglichkeit, in ihm die Niederschrift eines Vortrags, einer Rede zu sehen ${ }^{x}$. Zu diesem Hauptgrunde kommen einige kleinere Beobachtungen,

x In The Expositcr III hat C. Clemen den Versuch gemacht, Hebr. 3, I-4, 6 
die an sich nicht ausschlaggebend wären, aber mit ihm zusammengenommen unsre Hypothese wohl zu stützen imstande sind. Auch Wr. hat, wie cben sclion erwähnt wurde, auf das "wir" hingewiesen, das die ganze Schrift durchzieht. Noch bemerkenswerter ist der Wechsel zwischen der Anrede "ihr" und der Zusammenfassung des Schreibers oder Redners mit seinem Publikum durch den Gebrauch der I. Person Plur. Besonders auffallig ist dieser Wechsel in 3,12 ff. 6,9-12, 10, 24-29. Für einen wirklichen Brief wäre er nicht wunderbar (vgl. Röm. 6, 15 f. 7,4 ff. Eph. 4, I-16 u. a.) Aber grade für cine Epistel, für cinen literarischen Brief bleibt er etwas Unnatürliches und Sonderbares. Wir finden ihn in ähnlicher Weise im II. Clem. wieder, der einzigen Schrift jener älteren Zeit, - dies Wort in weiterem Sinne gefabt, die unstreitig eine niedergeschriebene Rede ist. Und es ist psychologisch leicht erklärlich, dab der Redner, ob er vor oder nach dem mündlichen Vortrag die Niederschrift vorgenommen oder veranlabt hat, sich der Situation, in der er vor seinen Zuhörern steht, vergegen!värtigt. Ferner könnte man zu der Stelle, die Deibmann hervorhebt, 13, 22, in der

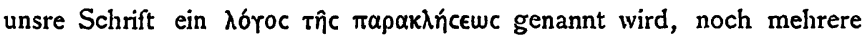
andre hinzufügen, in denen der Verfasser den gleichen Ausdruck anwendet oder seine Tätigkeit als ein $\lambda$ éreıv bezeichnet: 5, I I. 6, 1. 8, I. 9, 5. II, 32. Auch hier braucht man nur an I. Cor I, 6. 7, 8, Röm I5, 8, 2 Cor I, I8 und viele andre Stellen zu denken, um zu erkennen, daß diese Bezeichnung auch in einem wirklichen Briefe und selbst in einer Epistel nichts besonders Auffälliges an sich hätte. Wohl aber ist wenigstens an einigen dieser Stellen die Verbindung, in der jene $\mathrm{Be}$ zeichnungen gebraucht werden, derartig, daß sie für den mündlichen Vortrag natürlich und passend, für eine Abhandlung schwerfällig und umständlich erscheinen müssen. Dạzu gehört gleich die erste Stelle

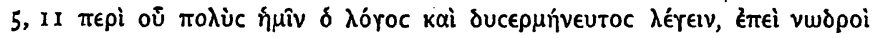

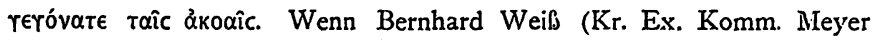
I 888 S. 140) von diesem letzten Worte sagt, es bezeichne die Sphäre, in welcher ihre Trägheit in Betracht komme, ihre Gehörwerkzeuge,

als eine den Zusammenhang unterbrechende Homilie zu erweisen; ferner hat sich S. A. Simcox in The Expository Times (1905?) ro dafür ausgesprochen, dab C. I-12 eine Homilie mit einer von Paulus angefügten Empfehlung sei; die Notiz über Timotheus in 13, 23 sieht er als in derselben Situation wie 2 Tim. 4, 9 geschrieben an. Beide Aufsätze haben mir nicht zur Verfügung gestanden; ich zitiere sie nach Th. J. B. Bd. 6 I, S. 146 u. Bd. 19I, S. 157. Aus älterer Zeit ist 2 u erwähnen J. Berger, der 1797 in der Göttingischen Bibliothek der neuesten theologischen Literatur B. III S. 449 einen Aufsatz: Der Br. an die Hebr. - eine Homilie, veröffentlicht hat. 
natürlich in geistigem Sinne, so ist das ja selbstverständlich richtig; von leiblicher Schwerhörigkeit oder Ermüdung in Hören hat er nicht reden wollen. Aber es ist doch bemerkenswert, daß er ein Bild gebraucht, das dem Vortragenden näher liegen mußte, als dem an ein ideales Publikum denkenden Schriftsteller. Vielleicht könnte man einwenden, daß ihm das Bild nahe lag, nachdem er einmal die Worte

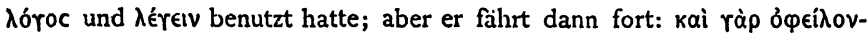

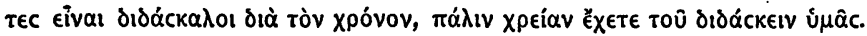
Mir scheint, als ob Wr. (S. 32) es sich doch zu leicht macht, wenn er meint, daß der Ausdruck "Lehrer sein" nichts weiter besage, als daß die Leser reif sein sollten in der christlichen Erkenntnis. „Hat man die Reife, so ist eben die Vorbedingung zum Lehren da: in diesem Gedanken hat der Verfasser seine Wendung gewählt." Man wird doch fragen dürfen: Welche Vorstellung hat er und haben denn die Leser oder Hörer - damit verbinden müssen, wenn zu ihnen gesagt wurde:

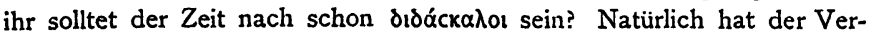
fasser nicht gemeint, daß sie alle sich zum Lehrerberuf vorbereiteten; er hat an diesem Ausdruck nur ihre christliche Erkenntnisreife messen

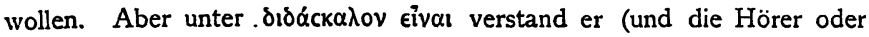
Leser) sicherlich nicht eine beliebige facultas docendi, sondern es verband sich für sie damit die Vorstellung der Lehrtätigkeit in der Gemeinde. Diese war aber so vorwiegend eine mündliche, lebendige und persönliche, daß die etwa beginnende literarische kaum in Frage kam. Ohne also hierauf so großes Gewicht zu legen, wird man doch sagen können, daß alle einzelnen Momente zusammengenommen, V. II u. I2 sich in den mündlichen Vortrag natürlicher und ungezwungener einfügen lassen, als in einen Brief, geschweige denn in eine schriftliche Abhand-

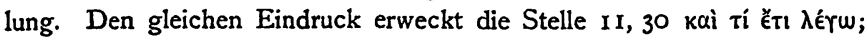

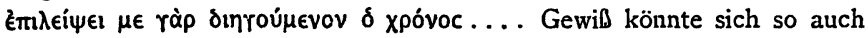
ein Schriftsteller oder ein Briefschreiber ausgedrückt haben. Aber auch dann müßte man sagen, daß er diese Wendung dem Magazin der Rhetorik entnommen hat, während der Redner auf seinem Gebiete geblieben wäre.

Freilich steht allen diesen Einzelbeobachtungen nun der Schluß der Schrift, offenbar nach anderer Richtung weisend, gegenüber. Die Worte C. 13, 22-24 lassen sich nicht anders verstehen, denn entweder als Teile eines wirklichen Briefes oder als Anhang mit der Absicht, der vorstehenden Abhandlung den Schein eines wirklichen Briefes zu verleihen. Und genau so wie bei der Erklärung, warum der Briefein- 
gang fortgefallen ist, ist man bei der Frage, wie dieser Briefschlub angefügt worden ist, auf Vermutungen angewiesen, die in der Schrift selbst keinen Anhalt haben. Man kann freilich darauf hinweisen, dal dieser Schlub als ein späterer Anhang deshalb erscheinen könnte, weil - vorsichtig ausgedrückt - einzelnes in ihm zum vorhergehenden nicht recht zu passen scheint. Wie ist der Verfasser nach seinen langen theoretischen Erörterungen, nach dieser Abhandlung, in der die lehrhaften Partieen den weitaus größeren Raum beanspruchen und nicht etwa (Wr. S. 16-20) bloß die Paränese vorbereiten sollen, dazu gekommen,

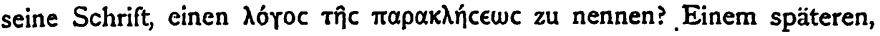
dem die Ermahnungen das wichtigste und wertvollste an der vorliegenden Schrift erschienen, lag diese Bezeichnung näher, als dem Verf. selbst. Ferner: ist er sich nach 5 , II dessen bewubt gewesen, dab er sein Thema ausführlich behandeln müsse, und nach I 1,32 dessen, dab er nachgrade schon fast $z u$ weitschweifig geworden sei, so ist die

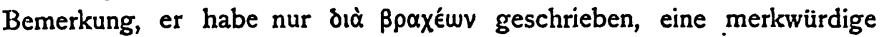
Entschuldigung. Endlich sei nochmals erwähnt, daß die Zusage seiner baldigen Ankunft in V. 23 immer wieder der Anlal für verschiedene Erklärungsversuche, warum er in solcher Voraussicht eine so lange lehrhafte Auseinandersetzung vorausschickt, geworden ist. Doch gebe ich $z u, d a b$ ein $z u$ starkes Betonen dieser Einzelheiten kleinlich oder pedantisch genannt werden könnte. Auch daß V. 20, 2 I einen natürlichen und sachgemäßen Schluß einer Rede bilden würden, ist nicht ausschlaggebend, da man nur auf I Petr. oder Phil. hinzuweisen braucht, in denen auch den feierlichen Schlußworten der Ermahnung bzw. Abhandlung noch einige persönliche Bemerkungen folgen. 'Wohl aber möchte ich noch auf folgendes aufmerksam machen, was für unsre Frage von Entscheidung sein könnte, weil es für die spätere Hinzufügung des Schlusses eine Erklärung zu bieten imstande ist. Außer den Schlußversen 13, 22 bis 24 wird nämlich für gewöhnlich auch 13 , I9 als Beweis für den Charakter des Briefes oder der Epistel angeführt; oder genauer das

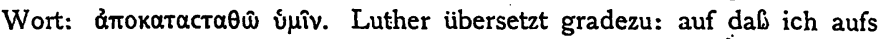
schierste wieder zu euch komme; Weizsäcker: damit ich euch rasch wiedergegeben werden möge. Das ist zwar vorsichtiger, wird aber wohl auch von ihm nicht anders verstanden sein, als von den meisten (soweit ich sehen kann, sogar, allen) Auslegern. Sie weichen mitunter in den von ihnen erratenen Gründen, warum der Verf. wiedergeschenkt werden soll, von einander ab, ob etwa Krankheit oder Gefangenschaft oder Verbannung oder andre persönliche Rücksichten ihn ferngehalten. 
haben; - aber darin sind sie einig, daß so nur einer sprechen könne, der von denen, die er anredet, räumlich entfernt ist. Tatsächlich aber braucht der Ausdruck an sich nicht zwingend zu dieser Annahme zu

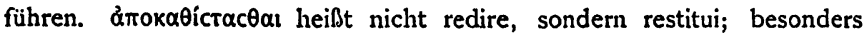
häufig wird das Wort in der medizinischen Literatur gebraucht in der Bedeutung: sich legen, aufhören - von Krankheiten, vom Fieber u. ä.; sonst heißt. es wiederhergestellt, in den vorigen Stand gesetzt werden, und wird von Personen überhaupt nicht gebraucht, auch im N. T. nur von Kranken Mc. 8, 25. 3, 5 u. Par. In einem Briefe würde man es unbedenklich mit wiedergesçhenkt werden im Sinne von zurückkehren übersetzen; aber d. i. nicht die einzig mögliche Übersetzung; an sich hindert nichts dies Wort und zwar in dieser Verbindung mit dem Dat. comm. auch in mündlicher Anrede zu gebrauchen. Für die Verhältnisse, Erwartungen oder Befürchtungen, die den Verf. zu diesem Ausdruck veranlaßt haben könnten, liegen mancherlei Möglichkeiten vor; und wir sind auf Vermutungen, aufs Raten angewiesen, aber nicht mehr, als wenn man die Worte einem von den Angeredeten entfernt Weilenden in den Mund legt. Es wird sich in beiden Fällen um den. Wunsch handeln, zu ihnen wieder in das frühere Verhältnis gesetzt zu werden, persönlichen Verkehr, Zusammensein und Gemeinschaft wiederzugewinnen; aber auch wer Abschied nimmt, wer nur vorübergehend an einem Orte weilt, wer vor einer ernsten Entscheidung steht, wem eine Gefahr droht, kann diesen Wunsch aussprechen und er wird keine Veranlassung haben, vor einem vertrauten Zuhörerkreise, dem die näheren Umstände bekannt sind, diese noch ausführlicher darzulegen. Auch von dem Schreiber eines wirklichen Briefes kann das gesagt werden, nicht aber von dem Epistolographen, der mit einer so leisen, verschiedene Auslegung zulassenden Andeutung nicht hätte zufrieden sein können. Gibt man aber die Möglichkeit zu, da $\mathrm{D}$. 19 auch von einem Redner im mündlichen Vortrag benutzt worden sein kann, dann ist es auch nicht mehr so fernliegend anzunehmen, daß grade dieser $V$. der Anla zur Hinzufügung des Schlusses geworden ist. Nachdem die Niederschrift erfolgt war, konnte in einer Zeit, wo man den ursprünglichen Charakter des Buches vergessen hatte, grade der Ausdruck ởrok. únîv zu der Vermutung verleiten, daß ein Brieftorso vorliege, den man wenigstens am Schluß wieder ergänzen wollte.

Freilich würden sich mit dieser Annahme neue Schwierigkeiten ergeben, die das Problem seiner Lösung nicht näher führen würden. 
Dic Hinzufügung eines Briefschlusses bliebe immer merkwürdig der Tatsache gegenüber, daß man nicht auch einen Eingang und eine Grußuberschrift fingiert hat; und besonders wenn der spätere Redaktor die Schrift 2u einem Briefe des Paulus hat machen wollen, aber auch in jedem andern Falle wäre es noch nicht etklärt, warum er sich nicht deutlicherer Hinweise bedient hat. Daher wird es doch geratener sein, diesem unsichern Boden der Hypothese des fingierten Schlusses, die sich cbensowenig überzeugend nachweisen läßt, wie die des verlorenen Briefeingangs, aufzugeben. Dann kämen wir aber nach allem vorangegangenen zu der Annahme, daß uns in Hebr. eine niedergeschriebene und dann an irgend eine Gemeinde versandte Rede vorliegt. Nur wird es notwendig sein, diese Schlubfolgerung zu modifizieren, und zu zeigen, daß sie - recht verstanden - nichts Absonderliches und Undenkbares an sich hat, sondern sich wohl in das Bild, das wir uns von der urchristlichen Zeit und ihrer literarischen Produktivität machen müssen, hineinfügt. Es sei zuvor noch einmal daran erinnert, dab wir in II Clem. ein untrügliches Beispiel dafür haben, da $\Phi^{\circ}$ auch damals Reden aufgezeichnet wurden und weiterhin zum Vorlesen dienten. Man hat sich nach Ergänzung dieser Schrift, soweit ich sehen konnte, ohne weitere Bedenken mit der Tatsache, daß uns hier ein "präliterarisches“ Produkt überliefert ist, nicht ein eigentlich literarisches Werk, abgefunden und sich auch nicht sonderlich gemüht, diese zunächst einzigartige Erscheinung $z u$ erklären. Auch Harnack hält die Versendung einer Predigt für ein Unikum, aber nicht für unmöglich, daß eine Predigt für wichtig genug befunden werden konnte, um sie einer Schwestergemeinde mitzuteilen, wobei sie natürlich mit einigen begleitenden Worten versehen worden sein müsse. Man müsse dann annehmen, „daß die Römer mit einigen kurzen begleitenden Worten diese bei ihnen gehaltene Predigt (eben II Clem.), weil sie ihnen besonders erbaulich und wichtig erschienen ist, an die Korinther gesandt haben, sei es, dab sie gebeten waren, ein Wort der Stärkung zu übersenden, sei es, daß sie es ungebeten taten." Mögen selbst mehrere Jahrzehnte beide Schriften von einander trennen, so wird doch, was man bei der einen erklärlich findet, auch der anderen zugestanden werden müssen. Es kann doch auch ein einzelner Lehrer eine von ihm gehaltene Predigt für wichtig genug gehalten haben, um sie $z u$ veröffentlichen oder einer anderen ihm nahestehenden Gemeinde $z u$ übersenden. Und was man gegen die Wahrscheinlichkeit

I Die Chronologie der altchristlichen Literatur bis Eusebius. 1897. S. $445 \mathrm{f}$.

29. 5. 1908. 
dieser Annahme sagen könnte, würde in demselben Maße auch für II Clem. gelten, für dessen Vorhandensein man mit Rücksicht darauf, da $B$ er sofort mit seinem Auftauchen als "Brief" vor uns erscheint, keine andere Erklärung, als die obige Harnacks, zu geben vermag.

Aber noch nach einer andern Seite hin läbt sich die Behauptung, Hebr. sei eine ursprünglich gehaltene und dann an eine Gemeinde versandte Predigt, ihrer Seltsamkeit entkleiden. Ich verweise noch einmal auf die oben erwähnte Äußerung Wredes, daß die in Io, $32 \mathrm{ff}$. enthaltene Mahnung von dem Verfasser schon vor der Niederschrift in mündlicher Predigt verwertet sein könne, und auf die ungefähr nach derselben Richtung weisende Bemerkung v. Sodens, daß uns in Hebr. ein Beispiel für die Art damals gehaltener christlicher Vorträge überliefert sei. Was Wr. von der einen Stelle zugibt, kann auch von anderen, auch von dem ganzen gelten, wenn man erst, wie auch er, erkannt hat, daf Hebr. mehr den Eindruck einer Rede, als den eines schriftlichen Erzeugnisses mache, - und v. Sodens Andeutung fordert gradezu zu der Schlubfolgerung auf, daß der Verf., wenn nicht seine Erörterungen überhaupt vorher schon mündlich vorgetragen, so doch sicher unter dem Einfluß seiner Eigenschaft und gewohnten Tätigkeit als Redner gestanden hat. Das aber wäre das eigentlich Wertvolle und Interessante für uns. $\mathrm{Ob}$ er die ausgesprochene Absicht gehabt hat, eine von ihm gehaltene Rede wörtlich aufzuzeichnen und andern mitzuteilen, kann uns im letzten Grunde gleichgiltig sein. Auch wenn man unsre Behauptung dahin reduziert, da $\$ der Verfasser nicht als meditierender Schriftsteller, sondern als Redner geschrieben, aus der Fülle des ihm in seiner Lehrtätigkeit naheliegenden Stoffes geschöpft und sich des hierbei von ihm angeeigneten Stiles bedient hat, bliebe sie nicht ohne Wert. Aber zwischen diesen beiden Polen gäbe es noch verschiedene Möglichkeiten. Wie weit und wo die unbewubte Benutzung rednerischer Form und rhetorischen Stiles zu einer beabsichtigten Rekapitulation schon verwerteter Gedankengänge und Ermahnungen geworden ist, darüber läbt sich nichts entscheiden. Mir will scheinen, als ob nicht bloß die lehrhaften Erörterungen, sondern grade die paränetischen Partieen der Schrift den Eindruck der Wiedergabe von mündlich vorgetragenem erwecken. Je weiter wir aber in dieser Richtung gehen, desto leichter erklären sich die Schwierigkeiten, von denen oben die Rede war. Sowohl das Fehlen des Briefeingangs, wie der Mangel eines konkreten Anlasses, wie endlich die wenigen, sich nur auf die letzten Verse beschränkenden Andeutungen des brieflichen Charakters werden verständlich, je mehr man 
annimmt, daß dcr Verfasser nicht ein Sendschreiben aus bestimmtem $A n l a b$ an eine ferne Gemeinde unter Berücksichtigung ihrer Verhältnisse, auch nicht cinem modernen Schriftsteller gleich eine Abhandlung für ein bestimmtes Publikum hat schreiben wollen, sondern dab er - „sei es, daß er gebeten war, ein Wort der Stärkung zu übersenden, sei es, $\mathrm{da} \cap$ er es ungebeten tat", - einem ihm nahestehenden Kreis von Christen das, was er anderen bereits vorgehalten hatte, auch zugänglich machen wollte.

An sich scheint diese ganze Frage von untergeordneter Bedeutung zu sein; wichtiger als die literarische Form einer Schrift und die Absicht des Verfassers nach dieser Seite hin, ist natürlich der Inhalt. Aber es läßt sich doch zeigen, daß sie - in den Zusammenhang der urchristlichen Probleme gestellt - für ihre Beurteilung nicht ohne Belang ist. Die obige Behauptung von dem unliterarischen Charakter jener ersten Jahrzehnte der christlichen Zeit wird kaum im Ernst geleugnet werden, wenn man zugibt, daß die größere oder geringere $Z$ ahl von Schriftwerken nicht ohne weiteres maßgebend ist. Selbst wenn, was kaum anzunehmen ist, uns nur ein kleiner Bruchteil aller schriftlichen Produkte jener Zeit erhalten geblieben wäre, müßte man doch zugeben, $\mathrm{daB}$ sie nicht in erster Reihe auf literarischem Wege wirken wollte. Es ist doch eben ein Kennzeichen enthusiastisch bewegter Zeiten, da $\$$ sie nicht in mühsamer Reflexion und angestrengter Spekulation sich einige literarische Produkte abpressen, sondern daß sich das Neue, das sie bringen, in einem gewaltigen Strome mündlicher Wirksamkeit ergießt. Man könnte vielleicht Lc. I, I-4 entgegenhalten; aber abgesehen davon, daß es sich bei den Evangelien um geschichtliche Stoffe handelt, liegt in diesen Worten noch keine Andeutung der Absicht, der Nachwelt die Kenntnis - des Lebensganges Jesu zu erhalten; andrerseits haben wir die Tradition, daß auch Mc. irgendwie auf mündliche Vorträge zurückzuführen ist. Sicherlich aber werden wir die Reste schriftlicher Zeugnisse aus solcher Zeit, - und zwar noch mehr, wenn wir der Überzeugung sind, daß sie höchstwahrscheinlich doch das wesentliche darstellen, was in ihr überhaupt geschrieben worden ist, - ganz anders ansehen, wenn wir uns dessen bewußt bleiben, daß sie nur ein schwacher Widerhall dessen sind, was sie bewegte. Sie sind nicht ein Codex aller Anschauungen, Einrichtungen und Verhältnisse, die man nur abzulesen brauchte, um über sie völlig informiert zu sein. Das wird nicht bloß in der praktischen Verwertung zu wenig beachtet; grade mit der historischen philologischen Kritik, der damit keineswegs ihr Recht abgesprochen werden soll, wird 
leicht die Neigung verbunden sein, in den schriftlichen Zeugnissen die Resultate peinlicher Überlegung, wie man den Stoff möglichst genau und umfassend überliefern könnte, anzusehen. Hier könnte wohl die religionsgeschichtliche Methode Wandel schaffen, wenn sie nur Ernst damit machen wollte, in den literarischen Erzeugnissen nur den Bodensatz unter den Wogen einer starken geistigen Bewegung zu erkennen. Es würde dazu dienen, wenn man versuchen würde, die urchristlichen Dokumente als einen Spiegel für die gewaltige mündliche Wirksamkeit jener Zeit zu betrachten und die Gesetze dieser Tätigkeit aus ihnen, soweit möglich, zu erschließen.

In besondern könnte uns Hebr. als ein Beispiel der lehrhaften Paränese geitten, für den $\lambda b_{\gamma}$ oc rvúcewc des Apostels Paulus (I Cor.

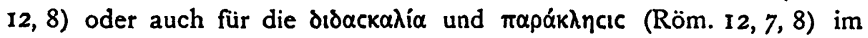
Unterschied von der pneumatischen Rede, der Prophetie, deren Vorbilder in den johanneischen Reden Jesu vorliegen. Für jene ist charakteristisch die typische Auslegung und Anwendung alttestamentlicher Begriffe und Anschauungen, die Verbindung von Lehre und Mahnung und die logische Entwicklung des Gedankens im Anschluß an ein bestimmtes Thema oder mehrere Themata, während die pneumatische Rede ihren Ausgangspunkt von einem kurzen Gleichnisworte nimmt, das dann von den verschiedensten Seiten aus beleuchtet wird, und die, ohne $\mathrm{da}$ b sie zu neuen Gedanken fortschreitet, in abwechslungsreichen Variationen fortfließt. Doch sind wir hier bei einer Frage angelangt, die nicht mehr zum Thema des literarischen Rätsels des Hebr. gehört und besondere eingehende Untersuchung verlangt. 